\title{
Éduquer ses veaux et génisses : un savoir empirique pertinent pour l'agroenvironnement en montagne?
}

\author{
Michel Meuret ${ }^{\mathrm{a}}$, Sabine Débit ${ }^{\mathrm{a}}$, Cyril Agreil $^{\mathrm{a}}$, Pierre-Louis Osty ${ }^{\mathrm{b}}$ \\ a Éco-zootechniciens, INRA, UR Écodéveloppement, Agroparc, 84914 Avignon cedex 9, France \\ b Agronome, INRA, UMR Agrosystèmes cultivés et herbagers, BP 52627, 31326 Castanet-Tolosan cedex, France
}

\begin{abstract}
Habituellement, c'est à la race qu'on attribue l'aptitude des bovins à pâturer en montagne. Pour des éleveurs laitiers du nord des Alpes, cette aptitude dépend d'abord de l'apprentissage acquis par leurs veaux et génisses vis-à-vis des fortes pentes. Ce décalage entre littérature et pratiques renvoie à la question bien peu référencée des pratiques d'éducation de l'animal. Ainsi, un nouveau contexte socio-politique, en l'occurrence la contribution du pâturage à la conservation de la biodiversité, peut être source de controverse et conférer une légitimité inédite à des pratiques ignorées par la recherche. Une telle situation montre également, comme s'y emploie souvent la revue, en quoi l'émergence de nouvelles relations natures-sociétés interpelle les sciences.
\end{abstract}

La Rédaction

\section{Mots-clés :}

pratiques d'élevage ; pâturage ; savoirs locaux; bovins laitiers; Alpes françaises

\section{Keywords:}

husbandry practices; grazing; local knowledge; dairy cows; French Alps
Résumé - La contractualisation du pâturage à fin de conservation de la biodiversité devient affaire courante, mais la question des pratiques appropriées demeure. C'est pourquoi nous avons enquêté auprès d'éleveurs revendiquant d'élever des vaches laitières compétentes pour le pâturage de terrains très pentus et embroussaillés dans les Bauges (Savoie et Haute-Savoie). Pour tous, indépendamment des races élevéeselles sont diverses -, l'aptitude des vaches à tirer parti de ces milieux résulte de pratiques d'élevage appropriées, bien plus que des caractéristiques raciales auxquelles se cantonnent les références en vigueur. Certains éleveurs veillent à apprendre spécifiquement à leurs jeunes bovins l'usage des fortes pentes. Ces pratiques d'apprentissage et la gamme des comportements évoqués incitent à s'interroger sur la «culture » des herbivores domestiques. Une perspective opérationnelle serait d'encourager ces pratiques et d'inclure dans les contrats agroenvironnementaux les espaces nécessaires aux apprentissages.

\begin{abstract}
Educating the herd's calves and heifers: an empirical knowledge usable in mountain agroenvironmental policies? Grazing contracts designed to protect biodiversity are becoming common fare although the question of appropriate practices has not yet been settled. This inspired the study, undertaken by the HERBIVORIE research network, of the impact of herbivores on plant dynamics in mountain areas. We interviewed herders who rear dairy cows which they claimed were perfectly able to graze on steep, bushy slopes in the Bauges mountains (northern French Alps). The survey was carried out using comprehensive interviewing techniques. Several reasons encourage the herders to graze their animals on steep slopes: urbanisation, gentler grasslands reserved for haymaking and dairy cows milked in the pastures, financial incentives to use grazing as a method to control scrub encroachment. Yet, current technical models discourage farmers from grazing non-controlled areas except with specialised breeds. We observed herders using steep uneven, bushy terrain for grazing dairy breeds (from the Holstein to the Tarine). All of them feel that the capacity to make best use of these lands can be traced to appropriate animal rearing practices, far more than to breed characteristics. Some herders are careful to teach their young cattle to use steep sloped lands. They say this is important because cows raised on lowland pastures are clumsy in the mountains and are accident-prone. They consider "education" to mean taking calves as young as 6-8 months old, and then heifers, to graze on special plots. These educational techniques and the behavioural diversity described by the herders raise the question of the "culture" of domestic herbivores and the role of herders in inter-generational transmission of the herd's skills. It may be profitable to encourage these practices and to include these learning areas in agro-environmental contracts.
\end{abstract}

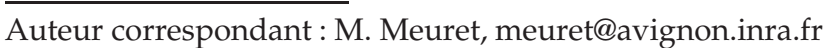


En Europe, en particulier dans les zones de montagne, les politiques territoriales impliquent de plus en plus l'activité d'élevage des grands herbivores pour mieux gérer l'embroussaillement et les accrus forestiers, entre autres conséquences de la modification des usages agricoles. Elles interpellent les écologues au sujet des interactions entre herbivores domestiques et populations d'herbivores sauvages, actuellement en développement accéléré. Elles les mobilisent également pour établir à présent des espaces naturels protégés sans exclure les usagers initiaux, dont les éleveurs. C'est dans cette perspective que des écologues de la faune sauvage et des dynamiques paysagères s'adjoignent les compétences de spécialistes des pratiques agricoles, afin de redéfinir ensemble leurs objets de recherche. C'est ainsi qu'émerge une écologie de l'action (Hubert, 2004), où les pratiques humaines deviennent constitutives des attributs et fonctionnalités des écosystèmes. Cette mutation des relations natures-sociétés engage une transformation des modes de production. Sur ces changements, leurs conditions et leurs enjeux, l'éclairage que les sciences peuvent apporter implique de connaître les modalités des pratiques concrètes en cause et d'en saisir les variations, sous l'influence conjointe des savoirs locaux et des politiques publiques.

Dans ce but, le réseau de recherche HERBIVORIE ${ }^{1}$ s'est investi dans la Réserve nationale de chasse et de faune sauvage des Bauges (Savoie et Haute-Savoie), en partenariat avec le Parc naturel régional (PNR), l'Office national de la chasse et de la faune sauvage (ONCFS) et l'Office national des forêts. Opérateur d'un grand site Natura 2000 sur les « hautes Bauges » (14 500 ha), le PNR affiche l'ambition de mieux conserver la biodiversité par le recours au pâturage des troupeaux domestiques. L'ambition est relativement courante, mais comment y répondre par des pratiques d'élevage appropriées? La question est posée, ici comme ailleurs (Agreil, 2003).

Or, nos premières observations chez des éleveurs ayant contractualisé du pâturage sur le site Natura 2000 (Encadré 1) ont révélé un comportement surprenant de génisses en alpage (Meuret et al., 2005). Celles-ci paissaient, en effet, paisiblement jusqu'au sommet des pentes et des éboulis, consommant volontiers les végétations très composites (Photo). Et ces éleveurs de surenchérir : «Nous, on élève des vaches-chamois, bien différentes des tartasses de plaine! » Cette interpellation nous a incités à considérer ces compétences originales, en tout cas peu référencées en élevage, et à en rechercher l'origine dans les pratiques des éleveurs. Serions-nous en présence de pratiques déjà très adaptées à l'agroenvironnement? Nous

\footnotetext{
${ }^{1}$ Le réseau HERBIVORIE associe depuis 2002 une vingtaine de chercheurs écologues et agronomes de diverses appartenances : CNRS, Inra, Cemagref et universités de Lyon 1 et de Grenoble. Il est animé par Anne Loison (CNRS, UMR 5558) et Hervé Fritz (CNRS, UPR 1934).
}

Encadré 1. Faire pâturer pour restaurer l'habitat des tétras-lyres

Dans les Bauges, le pâturage est censé contribuer à restaurer l'habitat du tétras-lyre, un gallinacé sédentaire de montagne. Le déclin marqué de ses effectifs met en cause, outre certains sports d'hiver, l'embroussaillement excessif, notamment par l'aulne vert (Alnus viridis, Chaix), qui fait suite au moindre pâturage des pentes et à l'abandon des récoltes de bois. Les tétras-lyres aiment à se percher sur les arbres, mais le mâle exige également pour ses parades des aires bien dégagées, alors que la femelle dissimule son nid sous des buissons bas, mais à proximité immédiate de pelouses. Quant aux oisillons, ils ont à se nourrir principalement d'insectes et de myrtilles. L'habitat à établir ou stabiliser consiste donc en des formations végétales complexes, souvent transitoires en l'absence de pâturage. (D’après Meuret et al., 2005.)

présentons ici des résultats d'enquêtes réalisées auprès de 15 éleveurs exerçant sur les 6 communes incluses dans le site Natura 2000 ou limitrophes, reflétant la diversité des élevages des hautes Bauges². Un guide d'entretien individuel a été conçu selon la méthode de l'entretien compréhensif (Kauffman, 1996) (Encadré 2). Les entretiens ont été transcrits et des extraits en sont ici tirés.

\section{Les raisons d'utiliser les pentes, à l'encontre du paradigme dominant du pâturage}

Dans les hautes Bauges, comme dans la plupart des massifs alpins où domine l'élevage bovin laitier, trois raisons incitent aujourd'hui les éleveurs à maintenir, voire développer, le pâturage des surfaces pentues, dans les alpages et aussi sur les flancs de vallées. Tout d'abord, la concurrence foncière due à la rurbanisation raréfie les terrains d'accès facile, alors même que les éleveurs ont besoin de toutes les surfaces mécanisables pour produire les foins nécessaires durant l'hiver. Les machines agricoles, conçues à l'origine pour les plaines, conduisent à concentrer l'intensification des prairies peu pentues. Cependant, la création de l'AOC fromagère Tome des Bauges impose de limiter l'usage des aliments concentrés et interdit l'ensilage ou l'enrubannage. En conséquence, les éleveurs adhérents sont incités à tirer un plus grand parti des ressources fourragères locales et notamment à étendre

\footnotetext{
${ }^{2}$ Ces enquêtes ont été réalisées dans le cadre d'une convention de recherche liant l'unité de recherche Écodéveloppement de l'Inra au PNR du Massif des Bauges (2004-2006). Elles ont tout d'abord donné lieu à un mémoire d'étudiant (Débit, 2005). La mise en débat locale et publique de leurs résultats a également conduit les auteurs à recueillir ensuite le soutien du Syndicat interprofessionnel de la Tome des Bauges, du Groupement de valorisation agricole des Bauges et du plateau de la Leysse, et de la Société d'économie alpestre de Savoie.
} 


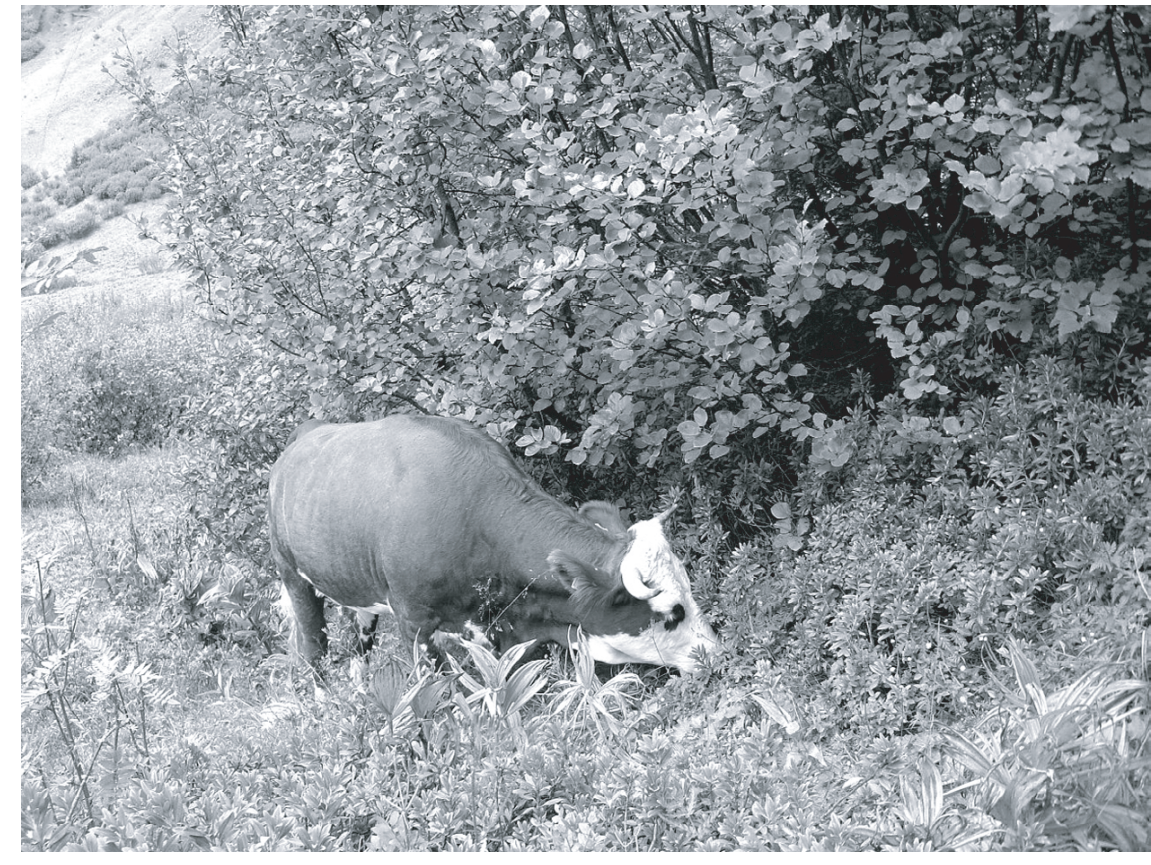

Photo. Génisse Abondance de 3 ans broutant en forte pente des végétaux trés divers sur un alpage classé Natura 2000. (Photo M. Meuret.)

\section{Encadré 2. Entretiens avec des éleveurs baujus}

Les entretiens individuels ont été menés auprès des éleveurs baujus ayant déjà, ou susceptibles d'avoir bientôt, des parcs d'élevage localisés sur le site Natura 2000 S15 des Bauges (alpages). Ils ont été conçus et conduits sur la base d'un guide d'entretien commun, consistant en une série organisée de fiches thématiques d'enquête (Kauffman, 1996), préalablement testées hors zone auprès d'interlocuteurs homologues. En 2 à 3 heures de dialogue, il s'agissait d'éveiller puis de soutenir l'intérêt de l'enquêté, en enchaînant des thèmes ayant à éclairer par des perspectives croisées et complémentaires une "question centrale ", objet de l'enquête, mais que l'on évitait d'aborder de front, hormis, et par honnêteté, lors de la prise de rendez-vous. Quinze éleveurs ont pu être enquêtés, puisque nous n'avons eu à essuyer aucun refus (Débit, 2005). Chaque entretien a donné lieu à une prise de notes, accompagnée d'un enregistrement avec retranscription intégrale.

Chacune des fiches fut composée de 4 sections : 1 . Un «mémo » pour l'enquêteur, rappelant l'objectif du thème ; 2 . Une « question de départ » concrète, quoique suffisamment ouverte (cf. infra) ; 3 . Des « attendus », anticipant les divers registres d'attitudes et de réactions probablement suscitées par la question de départ et incitant l'enquêteur à aborder ensuite une série de "questions de relance »; 4 . Les « questions de relance » visant à développer autour de la question de départ et, surtout, garantissant de stimuler le dialogue de façon similaire entre personnes enquêtées. L'utilisation des questions de relance a été ajustée au fil de chaque entretien, suite aux informations déjà obtenues.

La question de départ : «Je ne suis pas de la région, est-ce que pouvez me faire un petit historique de la ferme, pour m'aider à comprendre? » invitait très explicitement l'éleveur à choisir et à prendre le temps de développer d'entrée une réponse très personnelle. Les présupposés, ou valeurs parfois communément admises, notamment avec un enquêteur venu de l'Inra, furent anticipés et autant que possible évités. Il s'agissait même de tenter de surprendre, de brouiller les pistes sur «ce qu'on attendait ». Il s'agissait également de ne se faire assimiler ni à un contrôleur administratif de la PAC, ni à un technicien d'élevage, ni à un collègue éleveur très empathique.

Les thèmes des fiches successives étaient : 0 . Prise de rendez-vous par téléphone (car l'entretien démarre en réalité à ce stade) ; 1. Histoire de la ferme; 2 . Le troupeau aujourd'hui; 3 . La vie d'une vache ; 4 . Les alpages; 5 . Les pentes; 6 . Les broussailles; 7. Qu'est ce qu'une bonne vache? ; 8. Les choses à apprendre aux jeunes vaches?

les surfaces en pâturage. Enfin, les éleveurs sont également invités depuis peu à réinvestir les surfaces pentues plus en altitude, cette fois pour des raisons de conservation de la biodiversité 3 (PNR du Massif des Bauges,

\footnotetext{
${ }^{3}$ Des rémunérations leur sont proposées, depuis $60 € /$ ha pour la prime herbagère agroenvironnementale, jusqu'à plus de $400 € /$ ha lorsque, sous contrat d'agriculture durable en zone Natura 2000, ils doivent limiter l'importation en alpages des pratiques d'intensification sur prairies, mais aussi contenir l'embroussaillement par le pâturage.
}

2005). Ce sont des terrains pentus, embroussaillés et aux ressources incertaines, où le paradigme dominant pour la conduite des herbivores au pâturage, notamment des bovins laitiers, n'incite pas à s'aventurer (Chabert et al., 1998 ; Magda et al., 2001). L'enjeu étant spécifié, est-il tenable avec un outil - des vaches laitières au pâturage non originellement développé dans ce but?

Le paradigme dominant a fait ses preuves en prairies cultivées, depuis les admonestations de la Révolution fourragère de Chazal et Dumont (1955). Il tend à favoriser 
l'expression du potentiel productif des vaches sur des prairies dont agronomes et zootechniciens visent aujour$\mathrm{d}^{\prime}$ hui à optimiser le pilotage par des outils informatiques (Delagarde et O'Donovan, 2005). Il s'agit notamment de planifier, par un pâturage ras et uniforme, corrigé éventuellement par la fauche ou le broyage des refus d'herbe, la défoliation périodique d'une herbe à même de saturer sans effort pour la vache ses capacités d'ingestion et de digestion des nutriments. Ce mode d'alimentation s'apparente à la limite à des «auges de plein air ». C'est pourquoi les éleveurs deviennent plus férus de cultures fourragères et de machinisme agricole que de comportement de troupeau sur milieu végétal varié.

Ce paradigme domine quels que soient la région et le milieu. Il cadre les méthodes d'estimation de la qualité des pâturages (Agreil, 2003) et la définition des surfaces éligibles à la prime à l'herbe, une aide agricole attribuée à l'usage des surfaces exclusivement herbacées. On peut dire ainsi, en résumé, que le manuel du lycée agricole, la publicité dans le journal technique d'élevage et le formulaire de demande d'aide agricole convergent avec l'étymologie : un herbivore ne mange que de l'herbe. Pâturage est synonyme d'herbage, un bon éleveur tient ses parcelles "propres", c'est-à-dire exemptes de broussailles.

Mais les temps changent : en 2003, la prime herbagère agroenvironnementale (PHAE) a remplacé la prime à l'herbe et, dans la plupart des départements français, les pelouses partiellement embroussaillées, landes et sousbois, deviennent également éligibles. De ce fait, l'usage de parcelles "propres », qu'il soit effet de volonté, d'habitude ou d'obligation, entre en contradiction avec les attendus du pâturage à des fins de conservation d'habitats naturels. En effet, la plupart des espèces à protéger exigent pour leur survie le maintien d'un habitat que le paradigme dominant en élevage voue plutôt au gyrobroyeur (Encadré 1). Nous avons pourtant constaté que les éleveurs déduisent, même aujourd'hui, les portions embroussaillées de leur déclaration PHAE, tout en faisant pâturer ces milieux. Il est donc important de questionner l'état des connaissances au sujet des compétences de vaches sur milieux embroussaillés.

\section{La race des vaches laitières détermine en principe l'aptitude à la montagne}

La «valorisation des milieux d'élevage », dont la montagne, fait partie des aptitudes identifiées et évaluées par le Bureau des ressources génétiques (BRG), dans sa mission d'aider à la gestion et à la conservation de la diversité génétique des races. Institution multipartite fondée en 1983, il a largement recensé les aptitudes de races (BRG, 2004), au-delà des 6 races constituant plus de $80 \%$ du cheptel bovin national et focalisant l'attention des sélectionneurs.

Avec les 4 races de vaches laitières élevées actuellement dans les hautes Bauges, tout le gradient recensé par le BRG en matière de niveau de production et d'aptitudes à la montagne est représenté : depuis la race Holstein très répandue ( $67 \%$ des effectifs nationaux) et ajustée aux régimes alimentaires maïs-soja, jusqu'à la race Tarine (ou Tarentaise, 0,3\% des effectifs nationaux), montagnarde peu exigeante. Entre ces extrêmes, sont aussi élevées dans les hautes Bauges les races Montbéliarde et Abondance.

La Holstein, dont les aptitudes majeures sont «quantité de lait » et « vitesse de traite», sera exclue en 2007 de l'AOC Tome des Bauges afin d'éviter une banalisation du lait. Les 3 autres sont reconnues « races de montagne » par le BRG. La Montbéliarde est considérée comme rustique (Charron, 1988; Babo, 1998), car « capable de s'adapter à des conditions de vie variables ». Le BRG reconnaît des aptitudes supplémentaires à l'Abondance et à la Tarine : «adaptation à la marche » et "efficacité à la consommation de fourrages grossiers », et mentionne l'adaptation à la "vie en alpages ", grâce aux sabots de couleur noire plus résistants à la marche.

En montagne, l'endurance à la marche conditionne la performance laitière de la vache. C'est pourquoi l'Inra a évalué cette aptitude chez la Holstein, la Montbéliarde et la Tarine. Au terme d'essais sur une dizaine de kilomètres de marche, cette dernière s'est montrée la plus endurante. Les vaches ayant été élevées ensemble et ayant effectué les tests dans les mêmes conditions, les auteurs ont conclu qu'il s'agissait bien là d'une aptitude de race (Coulon et Garel, 1996; Coulon et Pradel, 1997). Des auteurs nordaméricains ont également évalué les aptitudes au déplacement des vaches, mais cette fois dans des pentes. À partir d'une synthèse bibliographique, Bailey et al. (1996) ont tout d'abord conclu que plusieurs grands herbivores, telles les vaches, évitent en général de pâturer des pentes supérieures à $10 \%$. Bailey et al. (2001) ont observé ensuite, dans des parcs d'élevage comportant des pentes de 0 à $55 \%$, que les Tarine en race pure ou croisées $1 / 4$ Hereford $\times 3 / 4$ Tarine montaient plus en altitude et en pentes plus raides que les Hereford pures ou croisées Hereford $\times$ Tarine. Enfin, Bailey et al. (2004) ont aisément distingué au sein des troupeaux les bottom dwellers (campeuses de bas de pente) des hill climbers (grimpeuses), non découragées par des pentes de 35 à $55 \%$. Ils recommandent de sélectionner sur ce caractère, afin de "valoriser au mieux les pâturages au relief accidenté ». Si leur proposition réussit et fait école, une nouvelle génération de vaches "grimpeuses ", parmi les croisées Tarine $\times$ Hereford, illustrera le fait que la pression de sélection est à conjuguer dans la durée avec la part d'héritabilité des compétences attendues dans un contexte d'élevage particulier (Audiot, 1995; Vissac, 2002). 


\section{Les compétences attendues des vaches dans les hautes Bauges}

Pour les éleveurs enquêtés, quelle est donc la « vache idéale »? Leurs avis convergent : c'est une vache qui, une fois sur l'alpage, "n'a jamais de problèmes de pieds ", «n'est pas trop lourde», « est dégourdie pour aller chercher l'herbe», «passe partout », et «produit du lait, bien sûr ! » Elle doit se montrer sûre dans les pentes, car c'est une question vitale, notamment en période de mauvais temps : «Par temps clair, tu les vois monter, donc tu vas les réceptionner. Tandis que dans le brouillard, tu ne vois plus tes vaches. Et là, elles montent, elles montent. Et, soit elles font demi-tour, soit elles vont trop en avant, elles glissent sur les dalles et elles vont s'écraser dans les pierriers. Et là, elles meurent! » Au quotidien, la compétence à grimper commande l'alimentation et, par là, la production: "Celles qui ne montent pas, elles vont continuer à ronger là où les autres ont déjà mangé [...]. Une vache, si elle ne sait pas grimper, elle n'est pas en état, donc ça va agir sur le lait.»

Cette compétence est recherchée, notamment par les

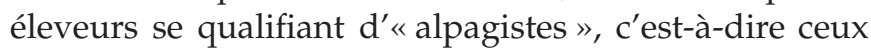
(12 sur les 15 enquêtés) menant durant l'été toutes leurs vaches laitières en alpage. Ces dernières sont alors confrontées à de fortes pentes ${ }^{4}: 25$ à $30 \%$ de moyenne, jusqu'à $45 \%$ chez 4 éleveurs : « La partie gauche de [notre] alpage est très pentue, on y a déjà perdu 3 vaches et une dizaine en sont redescendues esquintées. Déjà nous, pour se tenir, il y a une partie, c'est à 4 pattes que l'on y monte pour clôturer!»

Au vu des travaux scientifiques comparant les aptitudes des races, on pourrait s'attendre à trouver une proportion croissante des races Tarine et Abondance dans les troupeaux des Bauges ayant à utiliser de fortes pentes. Or, il n'en est rien. Les alpagistes dont les pentes sont les plus raides élèvent aussi bien des troupeaux composés des 4 races en diverses proportions et parfois croisées que des troupeaux de race pure Tarine aussi bien que Montbéliarde. Toutefois, les 2 éleveurs qui passent l'été en vallée ou sur un alpage peu pentu ont un troupeau constitué en majorité de Montbéliarde.

Tous les éleveurs enquêtés s'accordent à dire que les compétences attendues se trouvent non pas tant chez telle ou telle race, mais avant tout auprès d'animaux élevés chez eux ou dans les mêmes conditions. Ils tirent leur principal argument de comparaisons soigneusement faites chez eux entre des vaches ou des génisses élevées dans leurs troupeaux et d'autres, de même race, mais achetées adultes et le plus souvent en provenance

\footnotetext{
${ }^{4}$ Calcul des pentes réalisé par nos soins et ceux du PNR du Massif des Bauges. Les moyennes ne tiennent pas compte des zones plus plates très localisées, servant de "reposoirs " aux vaches. Rappelons ici qu'un col «hors catégorie » pour cyclistes correspond à une pente de 9 à $12 \%$.
}

d'élevages de plaine : «Déjà quand [les Montbéliarde achetées] sont arrivées [en bâtiment], elles étaient un peu paumées. Mais quand elles sont montées en alpage, c'était encore pire! [...] Elles restaient au pied de la montagne. Il n'y avait rien à faire, si tu n'étais pas trois personnes pour les monter [...], même avec le chien, elles ne faisaient que redescendre. » Un éleveur évoque une bête s'étant arrêtée in extremis après avoir glissé dans la pente : "C'était une qu'on avait achetée, qui venait de la plaine, qui était pataude comme tout, qui avait même le vertige, on aurait dit, dans la pente. Elle n'était vraiment pas dégourdie! » Un éleveur dont le troupeau est composé des 4 races depuis longtemps affirme : «Une Montbéliarde élevée chez nous, c'est comme une autre race, elles sont autant dégourdies. [...] Les Montbéliarde qu'on élève, elles grimpent tout autant que les autres. On a eu des Noires [Holstein], c'est pareil! Avant, on a élevé 2 ou 3 Noires, et elles grimpaient comme les autres. Mais si tu achètes une Montbéliarde [adulte], elle ne grimpera pas comme les autres, ça non!»

Ces observations et expériences empiriques conduisent certains éleveurs à utiliser le terme d'éducation : "D'où l'intérêt dans notre système d'élever en fait ses génisses. C'est une éducation que tu fais, du coup, depuis tout petit. Elles se forgent leurs pieds, leurs aplombs, leur musculation. Il y a tout qui s'adapte, quoi!» Avec un peu d'ostracisme montagnard, ils n'hésitent pas à dénigrer les vaches élevées en plaine : «Nous, on n'a pas le même système de vaches qu'en bas. Bon, en bas, ils ont de l'ensilage, puis c'est des bêtes, elles sont très rondes, elles ont à la limite de la cellulite sur la cuisse. Nous, c'est des vaches qui sont plus musclées, plus fermes, moins grosses. Ça n'a pas du tout la même allure!»

Toutefois, des éleveurs reconnaissent la prégnance des origines génétiques de leurs vaches, tant pour rejeter que pour préférer les unes ou les autres. D'une part: «Les Noires, c'est pas des bêtes pour nous!» «Elles redescendent [d'alpage] comme des squelettes, car on ne peut pas les complémenter comme il faudrait. Là où on les a [achetées], on a pris un bon niveau génétique, elles auraient fait facilement 8000 à $9000 \mathrm{~kg}$ [de lait]. Elles passent la journée entière à bouffer. Elles font le maximum de lait qu'elles peuvent, en puisant sur leurs réserves, donc ça fait un lait limite. » D'autre part : «En alpage, [la Tarine] c'est la bête par excellence! Même avec une Abondance, ça n'a rien à voir [...] au niveau de l'intelligence qu'elles ont pour aller se placer, pour aller se nourrir. »

\section{De l'éducation des vaches dès leur jeune âge}

Selon les éleveurs, «c'est le pays qui fait la bête». Mais, plus que l'altitude, le froid, le vent ou l'alimentation, 


\section{Encadré 3. « Les petits veaux ont l'avenir devant eux»}

La vie d'une vache laitière type des hautes Bauges peut être décrite comme suit.

C'est en automne, de préférence, que naît le "petit veau », au pré ou à l'étable. Il est aussitôt et définitivement séparé de sa mère, une fois tété le colostrum riche en défenses immunitaires. Il est installé en box individuel ou collectif et nourri au biberon de lait jusqu'à son sevrage, à 2 ou 3 mois. Mais, dès les premiers 15 jours, il va « apprendre à consommer du foin, quitte à en gaspiller beaucoup ». Il doit en effet développer au plus vite sa panse, c'est le gage d'une meilleure valorisation des fourrages durant le reste de sa vie. Ce veau est précisément une velle, les veaux mâles étant vendus vers 2 et 3 semaines.

Au printemps, âgé d'environ 7 mois, le veau sort pour la première fois. Il découvre la route, la clôture et l'herbe à brouter sur un petit pré proche de la ferme. Il passe avec ses contemporains le printemps et l'été en vallée, sur un ou plusieurs petits prés à proximité de la ferme, avec " un peu de foin ou de bonne paille, en cas de météo défavorable », visité par l'éleveur au moins chaque jour.

À l'automne, à l'âge d'un an, le voici « petite génisse ». On la ménage, elle rentre tôt à l'étable pour l'hiver. Nourrie au foin, elle reçoit aussi le «petit lait», un résidu nourrissant de la fabrication du fromage.

Nouveau printemps : dès les premières pousses d'herbe, elle ressort en prairies, mais cette fois sur des «côtes », parcelles très pentues, non utilisables par les vaches laitières et leur quai de traite mobile. En juin, elle découvre l'alpage après « une montée parfois assez sportive » pour disposer, tout l'été, d'une portion d'alpage dont les pentes, ici aussi, excluent les vaches laitières et leur quai de traite. À la descente d'automne, elle retourne sur les côtes, puis à l'étable dès les premières neiges. Âgée de 2 ans, elle devient « grosse génisse».

Son troisième hiver se passe soit dans son étable, soit « en pension » en plaine, lorsque le bâtiment et le stock de foins ne permettent pas d'accueillir tout l'effectif du troupeau. Elle est mise en reproduction en cours d'hiver, par insémination artificielle. Gestante, elle suit à 3 ans le même « circuit » que l'année précédente, mais sera descendue d'alpage plus tôt, afin de mettre bas après ses 9 mois de gestation, et devenir ainsi « vache laitière».

Son premier vêlage est crucial : «On dirait qu'elles perdent leur rusticité, elles ne savent pas ce qui leur arrive, elles sont toutes perdues. » L'inclusion dans le troupeau de vaches laitières, un groupe d'inconnues pas vraiment accueillantes, est source de stress.

Inséminée à nouveau, idéalement 2 mois après le vêlage, elle est traite durant 300 jours environ. Sa vie est ainsi rythmée par les alternances des 6 mois d'hiver à l'étable, des sorties de printemps et d'automne sur les prés, et des 3 à 4 mois d'été à l'alpage.

N'ayant commencé à produire du lait qu'au terme de sa $3^{e}$ année (contre 2 en systèmes intensifs), elle vit longtemps : 8 à 10 ans, voire 15 à 20 (contre 6 à 7 seulement en systèmes intensifs), si « elle reprend bien le veau » à chaque fois et «fait sans voir le vétérinaire ses 4000 à 5000 litres de lait par an». Au terme de sa vie, elle aura produit de quoi commercialiser environ 4000 tomes. Une fois réformée, elle finit sa vie loin des Bauges, menée à l'abattoir par un marchand qui l'aura engraissée auparavant, et sa carcasse sera consommée dans le relatif anonymat des « viandes de bœuf d'origine française ».

ce sont des pratiques spécifiques qui apprennent «les bonnes manières » à leurs veaux et génisses, futures vaches laitières. Nous proposons ici une catégorisation de ces pratiques, tenant compte de la fréquence des mentions faites spontanément. Puis nous détaillerons la pratique d'éducation ${ }^{5}$ à pâturer en fortes pentes, inédite dans la littérature scientifique et d'élevage.

En élevage laitier, et contrairement aux élevages allaitants ou aux herbivores sauvages, les jeunes sont séparés

\footnotetext{
5 «Éduquer» ou «élever»? C'est au XVII ${ }^{\text {e }}$ siècle que, s'agissant d'enfants, le verbe «élever» entra en concurrence avec le sens «éduquer», plutôt qu'avec celui de "nourrir et soigner ». Puis, au XIX ${ }^{e}$ siècle, le terme «élève » fut employé par analogie pour désigner les animaux ou les plantes «soignés par l'homme ». L'élevage consiste à préparer de jeunes animaux afin d'en faire des adultes aptes à produire, ce qui le distingue de la "production animale» (Béranger, communication personnelle), dont l'objectif s'est focalisé au XXe siècle sur l'obtention de "produits animaux» (lait, viande, poils...), entraînant avec lui la relative perte de sens du terme «élevage» (Béguin, communication personnelle). Toutefois, les animaux de renouvellement des troupeaux (génisses, chevrettes, agnelles...) conservent souvent aujourd'hui le qualificatif d'élèves, notamment lors des enquêtes statistiques agricoles.
}

de leur mère dès le premier jour (Encadré 3) et, de ce fait, ne disposent pas de modèle comportemental adulte à mimer. On comprend donc l'exigence élémentaire, pour les éleveurs, de créer et entretenir un lien fort dès la naissance. Cette exigence est généralement prise en charge et, le cas échéant, abondamment explicité : «Il faut dire que du point de vue du caractère [des vaches], c'est l'éleveur qui donne le caractère. [...] Ça se fait dès qu'ils sont nés, dès que tu commences à leur donner à boire et tout. Ils sentent tes sentiments, tes émotions, si tu t'en fous ou pas. Ça, je suis persuadée qu'ils le ressentent et qu'ils te le redonnent après. [...] Il y a déjà à la base le caractère de la bête, plus toi qui viens dessus. Si tu es tout le temps en train de taper, de gueuler, tu n'en feras rien de tes bêtes. Si, quand tu arrives dans le pré, elles se sauvent à la course, c'est bon, tu as tout faux, il faut changer de métier! »

Les éleveurs nous ont décrit en détail les raisons et les modalités de 16 pratiques d'apprentissage. Trois catégories peuvent être distinguées en fonction des comportements recherchés :

- le dressage pour les opérations au contact des outils et des dispositifs de l'homme - le travail en commun 
en bâtiment, au sens de Porcher (2002) - concerne des comportements énoncés ici par ordre décroissant de fréquence : " accepter d'être attaché »; " monter en bétaillère »; «suivre à pied et à la voix »; " boire le lait au seau »; «monter l'escalier de la salle de traite mobile »; «venir se faire traire à l'alpage » ; « bouser avant de sortir» ;

- le dressage pour acquérir de l'autonomie à l'extérieur, soit : « respecter la clôture électrique et les limites des parcelles » ; «savoir boire à l'abreuvoir automatique» ;

- l'apprentissage par une mise en situation de nécessaire autonomie, sans intervention directe de l'éleveur, soit : «savoir brouter»; «pâturer dans les pentes »; « connaître les prés et les alpages »; " suivre les meneuses "; "s'habituer à l'extérieur et aux bruits »; "s'intégrer au troupeau de laitières »; " apprendre la hiérarchie du troupeau ».

Il peut paraître surprenant qu'il faille apprendre à brouter, un comportement considéré généralement comme inné. Pourtant, c'est une pratique signalée aussi avec des chevrettes (Meuret, 1993). Pour l'apprentissage aux pentes, les éleveurs sont unanimes pour dire que la condition de réussite est le jeune âge de l'animal : «Le fait d'être mobile [pour brouter], ça vient surtout du modèle d'élevage. Si elles sont habituées petit veau, si elles vont d'année en année en montagne, ça restera toujours. [...] Parce que nous, elles font le système, déjà du départ, en côtes, et après elles continuent. » La limite d'âge supérieure est fixée par le premier vêlage, qui intervient ici à 3 ans (Encadré 3) : "Jusqu'à 2 ans, il n'y a pas trop de différences. Mais si tu ne les habitues pas avant 3 ans, après, en laitières, elles n'auront pas l'instinct de monter. À partir du moment où elles ont le veau dans le ventre, elles commencent à peiner. Et à l'alpage, celles qui sont moins habituées iront moins dans les pentes les plus sévères. » Les éleveurs ont observé qu'une vache achetée adulte demeure en mesure d'apprendre, mais qu'il lui faudra au moins un an d'accoutumance, avec le risque, ainsi, de ne pas s'alimenter correctement et de maigrir excessivement : «Si la vache n'est pas habituée toute petite, elle perdra du temps. »

Parmi les 13 éleveurs concernés par les pentes, 4 utilisent le terme d'éducation pour désigner l'apprentissage des veaux et génisses, tandis que 9 considèrent $\mathrm{qu}^{\prime}$ «elles s'habituent toutes seules ». L'analyse des calendriers d'élevage et des parcellaires montre que tous conduisent leurs veaux et génisses sur des parcelles très similaires (pentes de 25 à $45 \%$ ), aux mêmes saisons et aux mêmes âges. Seule diffère la raison de leur pratique : pour les uns, une démarche explicite d'éducation précoce ; pour les autres, la conjonction de nécessités conduisant à réserver les parcelles les moins pentues à la fauche et à la traite des vaches au pré.

Lorsque les éleveurs attribuent une visée éducative à cette pratique, cette dernière se déroule en 2 étapes et mobilise des parcelles spécialisées, c'est-à-dire constamment allouées aux mêmes catégories d'animaux. La première s'adresse aux "petits veaux », âgés de 6 à 8 mois (Encadré 3), et consiste à les mener dès leur toute première sortie, fin avril, sur une parcelle pentue et accidentée. Chez certains, c'est une parcelle d'une dizaine d'hectares, comprenant à la fois "du plat » et "de la côte", du pré et des bosquets. Chez d'autres, la parcelle d'un seul hectare est formée d'un talus herbeux et raide où les veaux, rentrés chaque matin par le bas, sont invités à rejoindre une zone plate plus confortable sur le haut, où est placé l'abreuvoir. Dans tous les cas, la parcelle est proche de la ferme, ce qui permet une surveillance à distance, ainsi qu'une visite quotidienne. Cette étape se poursuit jusqu'à l'approche de l'hiver, sur des parcelles de même nature, avec parfois un retour le soir au bâtiment pour la distribution du foin. La seconde étape s'adresse aux «petites génisses » de 1 à 2 ans, puis aux « grosses génisses » de 2 à 3 ans. Durant le printemps et l'automne, elle mobilise des coteaux en vallée, parcelles plus éloignées, généralement pentues (30\% de moyenne), embroussaillées et pour partie boisées. Durant l'été, il s'agit d'un «alpage à génisses » ou d'une portion d'alpage leur étant réservée, car l'accessibilité et les pentes ne permettent pas $\mathrm{d}^{\prime} \mathrm{y}$ traire les vaches laitières ( $45 \%$ de pente moyenne). C'est alors aussi l'occasion d'apprendre aux petites génisses à être mélangées aux grosses, apprentissage social qui facilitera, un an plus tard, l'intégration, délicate, des «génisses prêtes à vêler » au troupeau de vaches laitières.

\section{Des vaches dont l'intelligence serait à cultiver}

Les vaches des hautes Bauges se distinguent évidemment des « objets technicisés » (Simondon, 1958; Micoud, 2003) que sont aujourd'hui les vaches « routinisées » des élevages industriels, où l'ampleur des dispositifs technologiques et des précautions sanitaires vise précisément à réduire et à contrôler l'expression de leurs comportements. Mais elles se distinguent également des «brouteurs écologiques ", type Highland Cattle, privilégiés dans les réserves naturelles au motif de leur aptitude à une quasi totale autonomie (Lecomte, 1995). Ici, la relation entre l'éleveur et ses vaches est forte et entretenue. Chacune a son nom, indépendamment de son identification réglementaire, elle est surveillée de près lors des périodes délicates, ses traits de caractère sont bien connus et utilisés au cours de sa vie. Surtout, et ceci devient une originalité, ses capacités d'apprentissage sont mises à profit par l'éleveur afin de favoriser non seulement la docilité pour la traite et les soins, mais aussi l'autonomie dans la circulation et l'alimentation en alpage, où les conditions parfois rigoureuses mobilisent, au dire des éleveurs, des formes d'intelligence indispensables. 
Très généralement, ce qu'un éleveur dénomme « intelligence » est avant tout affaire d'individualité : telle vache est "dégourdie », telle autre est "pataude», et ceci se retrouve dans les populations tant à petits qu'à gros effectifs (Preiswerk, 1995 ; Faye, 1996). Ce qui est original dans les hautes Bauges, c'est que l'intelligence est revendiquée comme résultant d'une éducation in situ des individus, primant sur les traits de race originaux : «C'est tout pareil, du moment que c'est élevé chez nous!» Et les «élèves ", terme ancien désignant les jeunes animaux d'élevage, reçoivent bien une éducation, étant mis en situation répétée d'apprentissage, à des âges déterminés, en vue d'acquérir un comportement spécifié.

La conception de cet apprentissage, au moyen notamment de parcs spécialisés pour les veaux et les génisses, peut être qualifiée d'innovation technologique. Cette pratique des éleveurs demeure à ce jour empirique, car non traduite en recommandation à valeur générale, ni localement ni au-delà. Elle valide les travaux scientifiques ayant pointé le rôle prédominant de l'expérience précoce chez l'animal, des travaux pour la plupart circonscrits à la question de la docilité lors des manipulations (Bouissou, 1992; Raussi, 2003). Elle est originale dans son ambition d'éduquer à l'usage autonome de milieux pastoraux complexes. De fait, nos enquêtes intéressent vivement BEHAVE (Provenza, communication personnelle), un réseau de recherche et d'enseignement universitaire nord-américain qui aborde de façon très originale la «culture » des herbivores domestiques ${ }^{6}$. Ce réseau tente de proposer des moyens d'infléchir la culture des herbivores d'élevage par des conduites appropriées des troupeaux (Howell, 2005). L'un des initiateurs du réseau, nutritionniste de l'animal, va jusqu'à envisager le recours au teaching et au training (Provenza, 2004) .

\section{Des perspectives pour l'agroenvironnement en montagne?}

Les vaches laitières des hautes Bauges sont-elles prêtes à répondre aux attendus agroenvironnentaux? Pour partie seulement. Leur compétence «à grimper dans les pentes » est nécessaire pour pâturer plus en altitude, là où une biodiversité remarquable est à mieux préserver. Mais, selon leurs éleveurs, elles grimpent « pour aller

\footnotetext{
${ }^{6}$ BEHAVE (Behavioral Education for Human, Animals, Vegetation and Ecosystems Management) regroupe des écologues et des nutritionnistes des animaux domestiques ainsi que des praticiens de l'élevage, avec l'ambition d'enseigner comment mieux tirer profit des connaissances sur le comportement des animaux en milieux complexes. Cf. http ://www.behave.net/ (consulté le 22/02/2006).

7 Il est à noter que Fred Provenza a été ranch manager avant d'être professeur de nutrition animale et co-initiateur du réseau BEHAVE.
}

chercher l'herbe », et de l'herbe seulement. Or, l'agroenvironnement requiert de s'attaquer également, par le pâturage, aux broussailles jugées néfastes aux habitats d'espèces à protéger.

Il s'agirait donc d'apprendre aussi aux vaches à diversifier leur régime alimentaire en y incluant des broussailles comestibles. Là, nos enquêtes retrouvent les discours stabilisés dans les milieux de l'élevage, qui disqualifient a priori les broussailles (Meuret 2005) : «Ça ne vaut rien ", "Ce n'est pas avec ça qu'on fera du lait! » disent les éleveurs, qui affirment : «Mes vaches ne mangent que de l'herbe! » On pourrait conclure que le paradigme des parcelles propres (cf. supra) s'impose ici comme ailleurs. Mais, en creusant la question, c'est plutôt un manque de curiosité qui apparaît : «Je ne regarde pas»; «Ça m'intéresse tellement peu». Nous avons donc convié des éleveurs à visiter avec nous certaines de leurs parcelles, et force a été de constater que leurs vaches et leurs génisses s'attaquaient volontiers à plusieurs sortes de broussailles, y compris sur des milieux riches en herbe. Après un : «Elles font ça pour jouer!», et constatant l'impact souvent étendu de ce «jeu», plusieurs nous ont déclaré : "C'est étonnant, je vais y regarder de plus près. » On doit espérer que cette curiosité, partagée avec les opérateurs Natura 2000 du PNR, ainsi que les conseillers d'élevage, suscite bientôt l'identification de fonctions d'apprentissage, non seulement dans des parcs en pente, mais aussi dans des parcs offrant des végétations incluant des broussailles comestibles.

Du point de vue de l'écologie animale et de la zootechnie, cette perspective incite à évaluer les conséquences d'une pratique d'éducation précoce sur les performances des vaches adultes en lactation : quelle persistance des comportements acquis en parcs d'apprentissage? Quelles sont les stratégies de déplacements et de consommation chez des vaches expérimentées? Quelle est l'incidence sur la qualité et la quantité de lait produit? Ces questions pourraient ainsi permettre de revisiter et de prolonger des travaux antérieurs : nous avons montré avec des chèvres laitières et des brebis combien des milieux embroussaillés se révèlent parfois de valeur alimentaire aussi bonne que des prairies (Meuret, 1989; Agreil et al., 2004). Nous avons repéré également la pratique des parcs d'apprentissage en élevage ovin allaitant sur parcours (Meuret, en cours de production). Plus largement, ces questions renvoient au rôle de l'éleveur dans la transmission intergénérationnelle des compétences au sein du troupeau.

Du point de vue de l'ethnoécologie et des sciences de l'action, ces compétences des vaches et leur éducation ouvrent sur la question des savoirs et des savoir-faire des éleveurs. Ce sont eux qui valorisent ces compétences et assurent cette éducation. Comment se construisent ces savoirs empiriques? Sont-ils transmis et comment? Et comment de nouvelles façons de faire se font-elles 


\begin{abstract}
Encadré 4. Des éleveurs confrontés aux nouvelles habitudes de leurs vaches laitières
Les éleveurs apprennent en observant leurs troupeaux et ils sont parfois surpris. Ce fut le cas des éleveurs estivant par contrat leurs génisses dans la Réserve nationale de chasse et de faune sauvage des Bauges. Leur lot de vaches en première lactation, après cette expérience, ne se comporte plus sur l'alpage à vaches laitières comme le reste du troupeau : «[...] elles disparaissent de la vue, en allant fouiller dans les arcosses [massifs d'aulnes verts]. » C'est un problème pour les éleveurs, car « elles ne reviennent plus à temps pour la traite et il faut même parfois aller les chercher ». Pour eux, qui exploitent cet alpage familial depuis des décennies, c'est une surprise, quasiment une vexation. Ces nouvelles habitudes prises dans la réserve, pentue et embroussaillée, mettent en cause ce qu'ils savent du rythme de circulation et d'alimentation du troupeau en journée, comme de la valeur du pâturage offert : «On ne sait pas trop ce qu'elles y trouvent, là-haut. » Les éleveurs se sentent remis en apprentissage.
\end{abstract}

place? Cette dernière question est d'importance majeure. D'une part, en Bauges comme ailleurs, les savoirs hérités, même s'ils sont connus et valorisés, ne répondent pas à toutes les exigences du système technique d'élevage actuel. D'autre part, en Bauges, ces savoirs ont l'originalité d'être peut-être aisément ajustables à la conservation de la biodiversité. Ceci en ferait des savoirs à encourager (Nakashima et Roué, 2002). L'enquête auprès des éleveurs a montré la diversité de leurs rapports à l'animal concernant ses compétences pour l'utilisation des alpages. Cette diversité suggère aussi une approche comparative de l'acquisition des savoirs des éleveurs. Les changements dans les troupeaux, incités notamment par les politiques agroenvironnementales, sont parfois déstabilisants (Encadré 4). Mais ils peuvent, avec la coopération des éleveurs, aider à comprendre la genèse et le renouvellement des pratiques dans un domaine d'activité qui doit être innovateur.

Ces questions de recherche devraient trouver l'appui des concepteurs des dispositifs agroenvironnementaux, intéressés à mieux établir ces pratiques d'élevage. Comment pourrait-on qualifier la compétence d'un troupeau, et non plus seulement cadrer des effectifs d'herbivores et des dates de présence sur les sites? La perspective est qu'un troupeau préalablement éduqué, au lieu de stationner sur les portions plus planes, au risque de surpâturer les herbes et d'y éroder le sol, soit à même de réaliser spontanément des circuits quotidiens incluant les zones pentues et contribue, en les broutant, à maîtriser des plantes envahissantes, seraient-elles des broussailles. Dès à présent, on pourrait envisager d'inclure à la contractualisation les parcs d'apprentissage des jeunes animaux et les parcs de demi-saison, voire d'hivernage en plaine, lorsqu'ils sont disposés sur des coteaux embroussaillés qui pourraient à la fois nourrir correctement et éduquer des lots de génisses laitières.

Les éleveurs des hautes Bauges éduquent-ils leurs veaux et génisses pour mieux gérer les alpages avec leurs vaches laitières? Se poser la question, c'est renouveler le regard, à partir d'une singularité, sur un fonds commun des activités d'élevage. La compétence des vaches au pâturage est inséparable du "savoir-élever » des éleveurs. L'un et l'autre sont des produits sociaux dont il faut comprendre in situ la genèse, le renouvellement et le développement.

\section{Remerciements}

La réalisation de l'enquête a été facilitée par Caroline Petite, technicienne au Syndicat interprofessionnel de la Tome des Bauges, et Philippe Mestelan, chargé de mission «Natura 2000 et gestion des milieux naturels » au Parc naturel régional du Massif des Bauges. Qu'ils soient remerciés pour leur appui, ainsi que les éleveurs pour leur excellent accueil. Merci également à Marie Roué, ethnologue au Muséum national d'histoire naturelle, et Pascal Béguin, ergonome au CNAM de Paris, pour leurs conseils, leurs critiques et leurs encouragements.

\section{Références}

Agreil, C., 2003. Pâturage et conservation des milieux naturels : une approche fonctionnelle visant à qualifier les aliments à partir de l'analyse du comportement alimentaire chez la brebis. Thèse de doctorat, INA P-G, Paris.

Agreil, C., Meuret, M., Vincent, M., 2004. GRENOUILLE : une méthode pour gérer les ressources alimentaires pour des ovins sur milieux embroussaillés, Fourrages, 180, 467-481.

Audiot, A., 1995. Races d'hier pour l'élevage de demain, Paris, INRA Éditions.

Babo, D., 1998. Races bovines françaises, Paris, France agricole.

Bailey, D.W, Gross, E.A, Laca, E.A, Rittenhouse, L.R., Coughenour, M.B., Swift, D.M, Sims, P.L., 1996. Mechanisms that result in large herbivore grazing distribution patterns, Journal of Range Management, 49, 386-400.

Bailey, D.W, Kress, D.D., Anderson, D.D., Boss, D.L., Miller, E.T., 2001. Relationship between terrain use and performance of beef cows grazing foothill rangeland, Journal of Animal Science, 79, 1883-1891.

Bailey, D.W., Keil, M.R., Rittenhouse, L.R., 2004. Research observation: Daily movement patterns of hill climbing and bottom dwelling cows, Journal of Range Management, 57, 20-28.

Bouissou, M.F., 1992. La relation Homme-Animal. Conséquences et possibilités d'amélioration, Productions animales, 5, 303-318.

BRG [Groupes pilotes Ruminants et Porcins], 2004. Base de données nationale France: situation des ressources génétiques bovinsovins-caprins-porcins 2003, Paris, BRG.

Chabert, J.-P., Lécrivain, É., Meuret, M., 1998. Éleveurs et chercheurs face aux broussailles, Le Courrier de l'environnement de l'INRA, 35, 5-12.

Charron, G., 1988. Les Productions laitières, 1. Les Bases de la production, Paris, Lavoisier. 
Chazal, P., Dumont, R., 1955. La Nécessaire Révolution fourragère et l'expérience lyonnaise, Paris, Le Journal de la France agricole.

Coulon, J.-B., Garel, J.-P., 1996. Aptitudes à la marche de vaches laitières de types génétiques différents : influence d'un exercice répété sur la production laitière, Annales de zootechnie, 45, 349-355.

Coulon, J.-B., Pradel, P., 1997. Effect of walking on roughage intake and milk yield and composition of Monbéliarde and Tarentaise dairy cows, Annales de Zootechnie, 46, 139-147.

Débit, S., 2005. Des veaux bien éduqués : enquêtes sur les pratiques d'élevage des futures vaches laitières ayant à pâturer des terrains très pentus dans le massif des Bauges (France). Mémoire de $2^{\mathrm{e}}$ année de master recherche, Muséum national d'histoire naturelle / INA P-G / Université Paris VII-Denis Diderot, Paris.

Delagarde, R., O'Donovan, M., 2005. Les modèles de prévision de l'ingestion journalière $\mathrm{d}^{\prime}$ herbe et de la production laitière des vaches au pâturage, Productions animales, 18, 241-253.

Faye, B., 1996. Relations Homme-Animal dans l'élevage laitier intensif de Bretagne (France) : proposition d'une typologie des éleveurs d'après le tempérament de leurs animaux, Antropozoologica, 22, 17-22.

Howell, J., 2005. Les vaches auraient-elles aussi une culture? Un éleveur du Colorado témoigne, Fourrages, 184, 579-582.

Hubert, B., 2004. Pour une écologie de l'action : savoir agir, apprendre, comprendre, Paris, Arguments.

Kauffman, J.-C., 1996. L'Entretien compréhensif, Paris, Nathan.

Lecomte, T, 1995. Gestion écologique par le pâturage : l'expérience des réserves naturelles de France, Montpellier, ATEN.

Magda, D., Meuret, M., Hazard, L., Agreil, C., 2001. Répondre à une politique de conservation de la biodiversité: le pâturage des brebis pour la maîtrise des landes à genêts, FaçSADe, 12 .

Meuret, M., 1989. Feuillages, fromages et flux ingérés. Thèse de doctorat, Faculté des sciences agronomiques de Gembloux, Avignon.

Meuret, M., 1993. Piloter l'ingestion au pâturage, Études et recherches sur les systèmes agraires et le développement, 27, 161198.

Reçu le 23 février 2006. Accepté le 7 septembre 2006.
Meuret, M., 2005. Ressources de parcours pour ruminants, in Théwis, A., Bourbouze, A., Compère, R., Duplan, J-M., Hardouin, J. (Eds), Manuel de zootechnie comparée Nord-Sud, Paris, AUF-INRA, 293-304.

Meuret, M., en cours de production. Le Maître d'école apprend à ses moutons à manger de tout. Film documentaire, EPPGHV, Paris.

Meuret, M., Agreil, C., Vincent, M., 2005. Des vaches au secours des tétras-lyres : restaurer par le pâturage des habitats d'espèces fragiles, INRA mensuel, 122, 21-24.

Micoud, A., 2003. Ces bonnes vaches aux yeux si doux, in Dubost, F., Lizet, B. (Eds), Bienfaisante nature, Paris, Le Seuil, 217-237.

Nakashima, D., Roué, M., 2002. Indigenous knowledge, peoples and sustainable practice, in Timmerman, P. (Ed.), Social and Economic Dimensions of Global Environmental Change, Encyclopedia of Global Environmental Change, Chichester, New York, John Wiley and Sons Ltd, 5, 314-324.

PNR du Massif des Bauges, 2005. Document d'objectif Natura 2000 (2005-2011). Site S15 «Forêts, prairies et habitats rocheux des massifs orientaux des Bauges ", tomes 1 et 2 + annexes cartographiques.

Porcher, J., 2002. Éleveurs et animaux : réinventer le lien, Paris, Presses universitaires de France.

Preiswerk, Y., 1995. Pour une ethnoculture de l'élevage : vaches et "généalogies" dans la mémoire collective, Antropozoologica, 21, 31-38.

Provenza, F.D., 2004. Twenty-five years of paradox in plantherbivore. Interactions and «sustainable " grazing management, 57th annual meeting, Rangelands, 25, 24-30.

Raussi, S., 2003. Human-cattle interactions in group housing, Applied Animal Behaviour Science, 80, 245-262.

Simondon, G.,1958. Du mode d'existence des objets techniques, Paris, Aubier.

Vissac, B., 2002. Les Vaches de la République: saisons et raisons d'un chercheur citoyen, Paris, INRA. 